\title{
Design of Medical Devices That Meet Contradictory Requirements
}

\author{
Len Malinin $^{1}{ }^{*}$, Alexey Borovkov ${ }^{2}$ and Alexandr Mikhaylov ${ }^{2}$ \\ ${ }^{1}$ GEN3 Partners, 20 Winthrop Square, Boston, MA 02110, USA \\ ${ }^{2}$ CompMechLab, St. Petersburg State Polytechnic University, St. Petersburg, 29 Polytekhnicheskaya ul., 195251, Russia
}

\begin{abstract}
Background: Today vitally important medical devices are expected to meet diverse and increasingly stringent requirements, in order to ensure their dependability in scenarios which can be life-critical. These requirements can often be contradictory in nature, presenting additional challenge to the designer.

Method of Approach: In this article, discussed are two approaches to address contradictory requirements to medical devices: resolving contradictions and advanced Finite Element Analysis (FEA) modeling. First, it is shown how the contradictions can be resolved by separating the contradictory requirements in space, in time, and amongst the elastic parameters of the material. The generated designs still need be validated first by advanced modeling. Two factors make FEA modeling of medical devices especially important: risks or difficulties in producing experimental data and unknown sensitivity of the design characteristics to different parameters of the device and factors in the human body. Discussed are the selection of the material models and boundary conditions and sensitivity of the results to the parameters of the model.

Resolving contradictions and FEA modeling should not be contraposed to each other; they work more effectively in tandem.

Results and Conclusions: These approaches were applied to the development of peripherally inserted central catheters (PICC) and retrievable blood clot filters. The generated novel designs were protected by US patent applications. The results obtained in LS-DYNA and other packages are compared with the available experimental data for the kink test and material response.
\end{abstract}

The presented approaches can be used in the design and optimization of medical devices subject to stringent requirements or with enhanced properties.

Keywords: Contradictory Requirements; Medical Devices; FEA Modeling; Central Catheters; Retrievable Blood Clot Filters.

\section{INTRODUCTION}

The design approach, discussed in this article, is presented in the context of two medical devices, peripherally inserted central catheters (PICC) and retrievable blood clot filters (installed in vena cava). Addressing the contradictory requirements plays the leading role for the catheters, whereas FEA modeling of the retrieval process was vitally important for the design of the blood clot filters. The developed designs are covered in several patent applications [1-4].

The two mentioned medical devices represent important segments of the medical device market.

\subsection{Peripherally Inserted Central Catheters}

The treatment of a chronic disease often requires repeated and prolonged access to the vascular system for, e.g., the administration of medications, blood products, nutrients and other fluids and/or to withdraw blood. To avoid the discomfort and other side effects associated with the repeated insertion and removal of a needle for each session, a semipermanent catheter (e.g., a peripherally inserted central

*Address correspondence to this author at the GEN3 Partners, 20 Winthrop Square, Boston, MA 02110, USA; Tel: 617 728-7013;

Fax: 617 728-7500; E-mail: leonid.malinin@gen3partners.com catheter, PICC, Fig. 1a), may be used. A PICC is a catheter that is inserted into a vein at a peripheral location, such as the arm or leg, and threaded through the vein to the chest in proximity to the heart.

In 2010, the U.S. market for vascular access devices (VADs) and accessories, dominated by companies such as Becton Dickinson, CR Bard, Covidien, Smiths Medical and

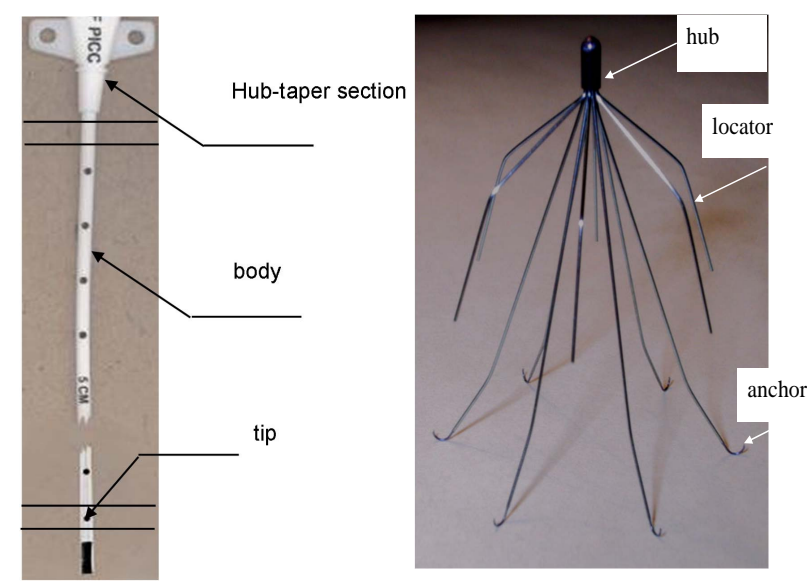

Fig. (1a). Peripherally inserted central catheter (PICC); b) Retrievable blood clot filter. 
Teleflex was valued at over $\$ 3$ billion, a $5.9 \%$ increase over the previous year. An important product category within VADs is represented by peripherally-inserted central catheters. PICCs first gained popularity in the US in the 1980s, becoming increasingly popular because of their reduction in cost and potential complications. Today they are used progressively more to deliver chemotherapy and other intravenous fluids and medications [5]. Moreover, clinicians are beginning to utilize power-injectable PICCs for patients who could possibly require imaging in the future. The leading PICC vendors include, in addition to listed above, companies such as Cook, AngioDynamics, Arrow (acquired by Teleflexin 2007), and Vygon. Growth in the market for powerinjectable PICCs has simultaneously increased the market for polyurethane PICCs, as power-injectable PICCs are predominantly constructed from polyurethane.

PICCs have several advantages over centrally inserted central catheters, such as options for bedside insertion by nursing staff and local anesthesia, and low risks of pneumothorax and major hemorrhage [6,7]. The major PICC parameters indicated by the vendors in the FDA submissions and operation manuals are maximum injection pressure and flow rate, as well as kink resistance. Injection rate has been the limiting factor for power injection with a constant maximum pressure rate. Usually injection pressure of 300 psi $(2,068 \mathrm{kPa})$ is specified as the limit, and the flow rate of 2 $\mathrm{mL} / \mathrm{sec}$ is often quoted as a safe value in tests, though rates of $5 \mathrm{~mL} / \mathrm{sec}$ and higher are possible [8-11].

Exceeding the maximum flow rate or the maximum pressure of power injection may result in catheter failure and/or catheter tip displacement. Designs optimized computationally or through solving inventive problems can reduce the probability of failure. These solutions are discussed in this article.

\subsection{Blood Clot Filters}

Inferior vena cava (IVC) filters are implanted into the vena cava to trap blood clots, preventing or reducing the likelihood of a life-threatening pulmonary embolism (PE). IVC filters can be made of stainless steel, titanium, or nickeltitanium (nitinol).

The IVC filters can be permanent or retrievable. Permanent filters, which are designed to remain in the patient without the ability to be removed, should ensure secure fixation to the vena cava wall to prevent migration of the filter. Retrievable filters are designed so that they can be removed from the vein with catheter-based retrieval devices when the risk of PE has subsided or when the patient no longer has a contraindication to anticoagulation therapy.

Most designs of IVC filters are collapsible cone-shaped arrays which comprise axisymmetrically arranged struts with hooks (barbs) on the wire ends to secure the filter to the vena cava wall, and locators, which position the filter in the vein (Fig. 1b). These filters have been designed for compression into a small size to facilitate introduction into a vascular passageway and subsequently expandable into contact with the walls of the passageway [12]. Permanent filters include a structure to anchor the filter in place within the vein, for instance, by elongate diverging anchor members with hooked anchors that penetrate the vessel wall. The hooks on the fil- ters of this type are relatively stiff and do not bend, and within two to six weeks after a filter has been implanted, the endothelium layer grows over the hooks and locks the filter in place. After that, any attempt to remove the filter results in a risk of rupture of the vena cava [12].

To those patients who have only a short term risk of pulmonary embolism, and therefore are averse to receiving a permanent implant, retrievable blood clot filters present a more attractive alternative. If such a filter is withdrawn before the endothelium layer grows over the anchor members, damage to the vena cava wall is minimized. However, after growth of the endothelium layer the combined inward and longitudinal movement of the filter sections as they are drawn apart can tear this layer.

Retrievable filters need to satisfy stringent requirements and still can lead to multiple complications. As of 2010, there were three retrievable filters approved by the Food and Drug Administration (FDA): 1) Gunther Tulip (Cook, Inc., Bloomington, Indiana), 2) Recovery Filter (Bard Peripheral Vascular, Inc., Tempe, Arizona), and 3) OptEase (Cordis Endovascular, Warren, New Jersey) [13]. In 2007, 167,000 filters were implanted, with a projection of approximately 259,000 filters being implanted in patients in 2012. This growth may be linked to the introduction of retrievable filters [14]. A significant complication, prevention of which is discussed below, is difficulty of filter removal, arising due to in-growth (endothelialization) of the filter.

\section{METHODOLOGY: RESOLVING CONTRADIC- TIONS. THE CASE OF THE PERIPHERALLY INSERTED CENTRAL CATHETERS}

\subsection{Single Lumen Catheter. Separating Contradictory Requirements in Space}

In many cases, the catheter must be able to operate in multiple modes, which presents strict requirements to its design and material. For instance, to simplify insertion and reduce discomfort, PICCs and other semi-permanent catheters are generally made thin and flexible, which limits their structural strength, and, in turn, the maximum pressure and flow rate a catheter can handle. This leads to contradictory requirements to the design and material of the device. For instance, in terms of the wall thickness, the catheter needs to be highly flexible in order to facilitate/enhance its penetration, and therefore the thickness of the walls and the moment of resistance of its cross-section should be low; however, the wall thickness needs to be high to increase the maximum pressure and flow rate during power injection. (The external diameter of the catheter is limited by the vein diameter and cannot be varied).

Another requirement to the catheter design is visibility under X-ray fluoroscopy, by which the location of the tip of the catheter is ascertained. To that end, a radio-opaque agent, such as $\mathrm{BaSO}_{4}$, is often introduced in the catheter material. However, presence of radio-opaque agents is known to weaken the catheter materials [15].

Likewise, exposure to alcohol or other solvents leads to degradation of such catheter materials as polyurethane [15]. A combination of these two factors $\left(\mathrm{BaSO}_{4}\right.$ and alcohol) can lead to a failure in the proximal part of the catheter, which is 
Table 1. Requirements to Different Parts of the Catheter

\begin{tabular}{|c|c|c|c|c|}
\hline & Proximal & Distal & \multicolumn{2}{|c|}{ ID } \\
\hline Flex test & 2 & 0 & 0 & 2 \\
\hline Alcohol resistance & 2 & 0 & 2 & 2 \\
\hline Tensile Strength & 2 & 1 & 0 & TBD \\
\hline Burst Strength & 2 & 2 & 2 & 2 \\
\hline Kink Resistance & 2 & 2 & 2 \\
\hline Visibility & 0 & 2 & 0 \\
\hline Chemical compatibility & 1 & 1 & 2 \\
\hline White color & 2 & & 2 \\
\hline
\end{tabular}

close to the peripheral location (e.g., on the arm) of the patient subject to disinfection by alcohol.

To summarize, different conditions that a PICC catheter should meet are listed in Table $\mathbf{1}$.

Based on these requirements, usually a compromise design is selected, providing, for instance, a certain level of flexibility and ability to handle a certain pressure, and likewise seeking a balance between other requirements. Such a design most often implies that all parts of the catheter must meet all requirements.

A more attractive way to address the contradictory requirements is based on the separation in space approach, one of the standard methods of resolving contradictions [16]. Indeed, as can be seen from Table 1, different requirements apply to different parts or segments of the catheter. The separation in space approach leads to design of a nonuniform catheter with superior characteristics. Such a catheter has respective segments with different properties, e.g., reinforced at the material level, or at the design level [1-4, 17].

For instance, the hub-taper section of the catheter (Fig. 1a) is most often damaged in tests and modeling. This is due to such factors as the boundary effect of the hub and stress concentration at the transition from the taper to the body, and deterioration of the material due to (hot) casting. Therefore, redesign of the hub-taper section has significant effect on the strength of the catheter. A few design steps can improve the strength of this area (reinforcement, optimal elasticity of the hub, cold casting). These recommendations were confirmed by FEA modeling (to be published).

\subsection{Single Lumen Catheter. Separating Contradictory Requirements in the space of elastic parameters}

Further analysis of requirements to a single lumen catheter leads to an interesting material optimization problem which arises from analyzing the burst pressure and kink test requirements.

When operating in the fluid transfer mode, a PICC must be able to hold sufficiently high pressure. The fluids, which

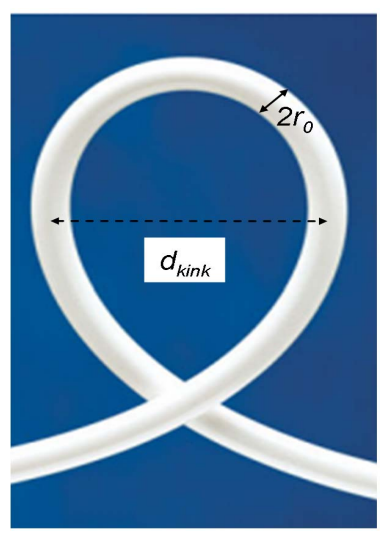

Fig. (2). Kink deformation of the catheter.

are infused through the catheter, are supplied from a pressurized source. The speed of infusion is important, as faster infusion reduces the time to administer a treatment and the cost of the procedure. However, infusing under pressure (which can reach 300 psi [2,068 $\mathrm{kPa}]$ ) demands sufficient strength of the catheter. Fluids escaping a catheter which has failed (e.g., after the maximum flow rate and/or pressure has been exceeded) may damage surrounding tissues or cause even graver consequences.

At the same time, the catheter is required to withstand the so-called kink test (testing the ability of the catheter to maintain an open lumen when it is bent at a radius appropriate for the intended anatomy [18]). The kink test (Fig. 2) reflects operational conditions when the catheter can be folded many times at the arm of the patient, and requires high elasticity of the catheter. Circumferential deformation $\varepsilon$ of the catheter during the kink test can be estimated as

$$
\varepsilon=\frac{\pi\left(d_{k i n k}+2 r_{0}\right)-\pi d_{k i n k}}{\pi d_{k i n k}}=\frac{2 r_{0}}{d_{k i n k}}
$$

where $d_{k i n k}$ and $r_{0}$ are diameter of the loop and radius of the cross-section of the catheter.

A catheter that can withstand both the kink test and high burst pressure is usually designed in a sequence of iterations 


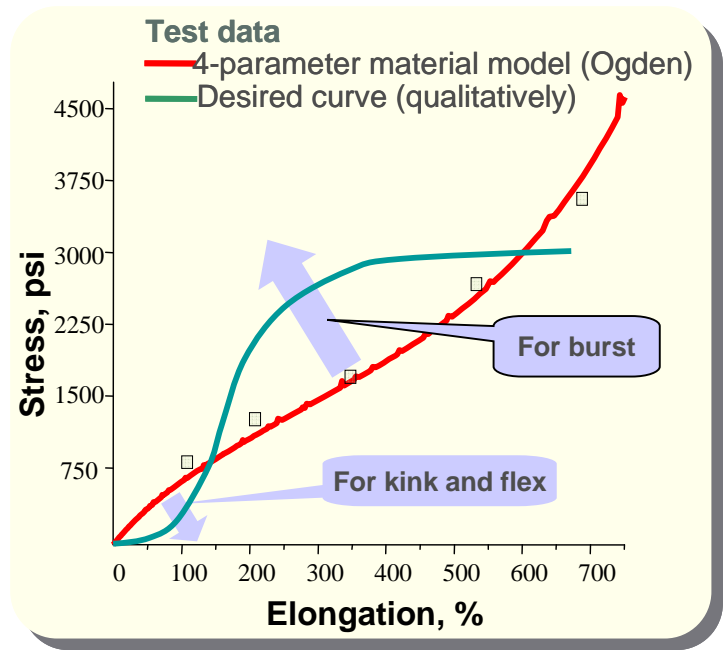

Fig. (3). Separating contradictory requirements in the space of elastic parameters ( $\mathrm{s}-$ stress, e - deformation).

Table 2. Deformation of 6-4F Catheter During Kink Test

\begin{tabular}{|c|c|c|c|c|}
\hline Catheter 6-4F & \multicolumn{2}{|c|}{ Tip } & \multicolumn{2}{c|}{ Body } \\
\hline \hline $\boldsymbol{d}_{\boldsymbol{k} \text { in } \boldsymbol{k}, \boldsymbol{m m}}$ & $\boldsymbol{r}_{\boldsymbol{0}}, \boldsymbol{m m}$ & $\boldsymbol{\varepsilon}, \boldsymbol{\%}$ & $\boldsymbol{r}_{\boldsymbol{0}}, \boldsymbol{m m}$ & $\boldsymbol{\varepsilon}, \boldsymbol{\%}$ \\
\hline \hline 6.985 & 0.673 & 19 & 1.00 & 29 \\
\hline 15.24 & 0.673 & 9 & 1.00 & 13 \\
\hline
\end{tabular}

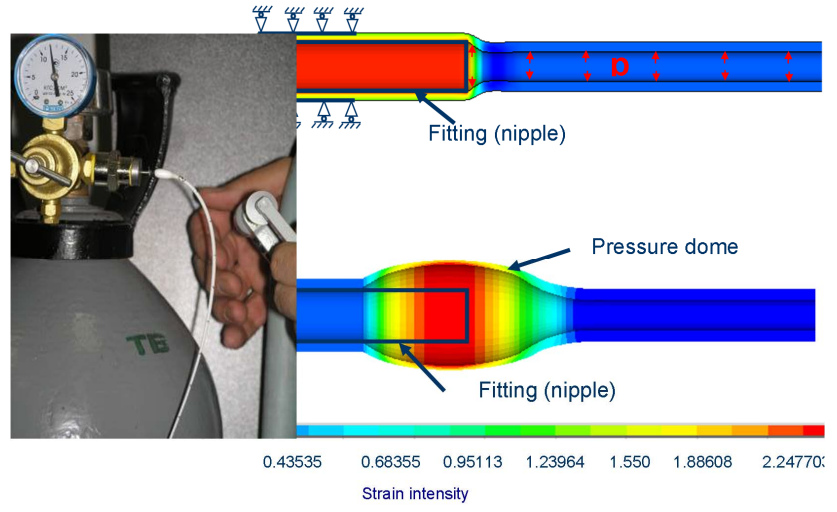

Fig. (4). Burst test of the catheter (deformations exceed 200\%).

that represent a trade off between the two contradictory requirements.

Different materials are known to perform better at one or the other test. E.g., comparing the two materials that have been used extensively in catheters, cross-linked silicone rubber and thermoplastic polyurethane (TPU), silicone catheters bend more easily, but kink with less applied force than polyurethane catheters.

However, this material optimization problem can be recast as identification of a catheter material having different properties, high elasticity (for the kink tests) and at the same time high strength (for the burst tests). This means that the target material should meet contradictory requirements to $a$ single characteristic, its stress-deformation curve.

Again, one should not expect that a material with uniform (linear) relationship between stress and strain will meet

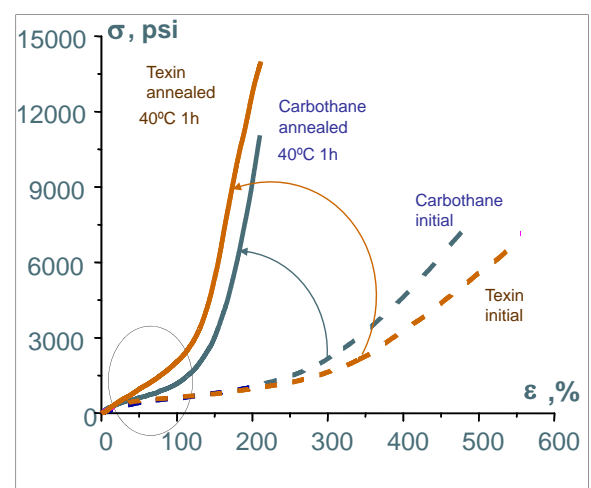

Fig. (5). Effect of annealing on elastic properties of texin and carbothane (annealing at $40^{\circ} \mathrm{C}$ for about 1 hour under uni- and bi-axial mechanical load).

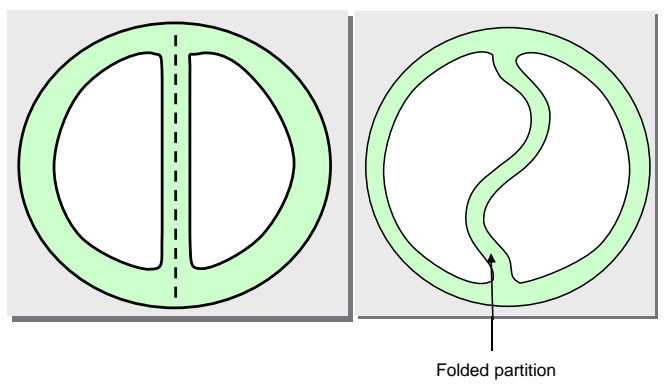

Fig. (6). Double lumen catheter with fixed (left) and folded (right) partition.

the requirements in both tests. The contradiction can be resolved using the fact that high elasticity (the kink tests) is required at relatively low deformations (Fig. 3 and Table 2), whereas high strength (burst tests) is required at large deformations. Based on experimental and analytical data (Fig. 4), deformations at burst exceed $200 \%$, whereas at kink deformations do not exceed 30\% (Table 2). Therefore, the requirements can be separated in the space of elastic parameters of the material (Fig. 3). The desired (non-linear) stresselongation characteristic would represent very elastic material at low deformations, toughening up as the deformations grow.

The material with such characteristic can be indeed designed. The driving factors, affecting the $\sigma-\varepsilon$ curves in the desired direction, include nano-fillers and thermomechanical treatment [3]. The latter is illustrated in Fig. (5), showing the effect of annealing on the $\sigma-\varepsilon$ curves of the two common catheter materials, texin and carbothane. As can be seen from Fig. (5), the $\sigma-\varepsilon$ curve of these materials after annealing takes a shape similar to the target shape in Fig. (3) (progressive stiffening as deformations grow).

\subsection{Double Lumen Catheter. Separating Contradictory Requirements in Time}

Conventional catheters have a single lumen for applying suction and for transporting fluids (such as therapeutic agents, nutrients, irrigation fluid, contrast media, and sc forth). Only one of these functions can be performed at a particular time, and single lumen catheters typically alternate between applying suction and providing fluid. The alterna tive to using a separate catheter is a catheter with two inter nal lumens (Fig. 6). Double lumen devices enable the flow o materials in opposite directions simultaneously. 


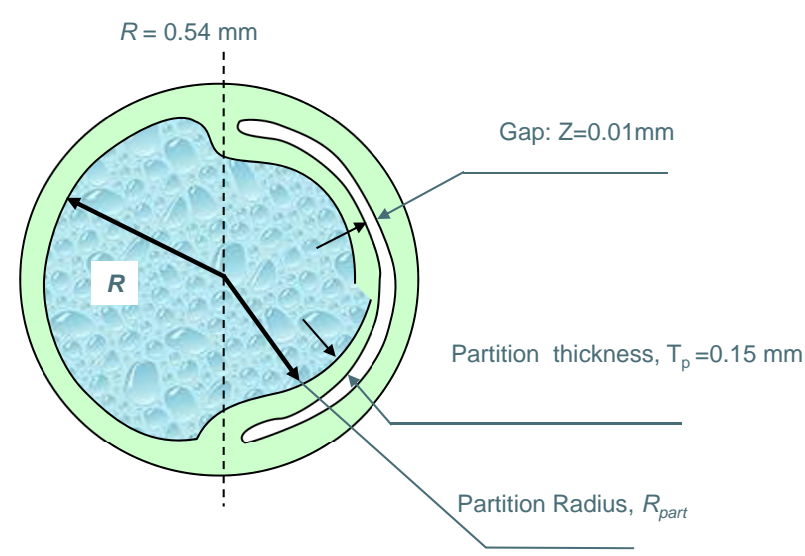

Fig. (7). Double lumen catheter with folded partition in power injection mode.

The fluid needs to be supplied through the catheter at high pressure and flow rate. For instance, contrast media requires a catheter capable to withstand the high injection pressures and flow rates necessary to disperse the media throughout an area of interest. These pressures and flow rates are often beyond the permissible ones for typical semipermanent catheters. While the safe rates quoted by vendors are typically in the $2-5 \mathrm{~mL} / \mathrm{sec}$ range, in-vitro ruptures at flow rates of 4 and $15.4 \mathrm{~mL} / \mathrm{sec}$ were reported for polyurethane PICCs and at $0.5-3.5 \mathrm{~mL} / \mathrm{sec}$ for silicon PICCs [19]. The maximum flow rate used for CT (computed tomography) arteriography may reach $17 \mathrm{~mL} / \mathrm{sec}$ [19]. It is therefore desired to develop the design for a double-lumen PICC, which can withstand a higher flow rate.

While non-uniform reinforcement of the catheter material, described above, is one of the options, the design of the two lumen catheter presents another option to improve strength. Indeed, the maximum flow rate is only needed during power injection, and power injection typically takes place through one lumen only. Because of the partition, the area of the other lumen is not available during power injection. Ideally, the cross section of one of the lumens should be maximal during power injection, and then return to a normal size under normal fluid delivery or removal. We therefore again come to a contradictory requirement, which can be stated as follows: the partition is needed in order to deliver different fluids, and the partition is not needed during power injection.

One of the known methods of resolving such a contradiction [16] is separation in time, which brings up the idea of the dynamic partition (Fig. 6) [1]. The partition unfolds under high pressure, effectively making the cross-section of both lumens available for transmission (Fig. 7). The increased cross-section can enlarge up to 16-fold the flow rate $Q$, which is proportional to $R_{\text {eff: }}^{4}$

$Q=\frac{\pi \cdot R_{e f f}^{4} \cdot \Delta p}{8 \cdot \eta \cdot l}$

where $R_{\text {eff }}$ is the effective radius of the cross-section, $D p$ is pressure gradient, $h$ is viscosity of the fluid and $l$ the length of the catheter (while the Poiseuille's law requires steady laminar flow, which may not be the case for power injection, Eq. (2) still serves as a useful approximation).

\section{METHODOLOGY: FEA MODELING. THE CASE OF THE PICC CATHETERS}

The designs proposed above were validated by extensive FEA modeling for the existing materials, of which we present two examples below:

- $\quad$ Kink test analysis: determine the critical radius of the loop corresponding to loss of stability (buckling) in the kink test and its dependence on the elastic properties of the material, and

- Power injection analysis: determine the maximum pressure that the double lumen catheter with a folded partition (Fig. 7) can withstand.

The target burst pressure that the catheter should withstand was specified as $300 \mathrm{psi}(2,068 \mathrm{kPa})$ and significantly exceeded the maximum pressure of the baseline design. At the same time, the observed diameter of the kink loop of the baseline design, $7.0 \mathrm{~mm}$, was below the minimum requirement, $15.24 \mathrm{~mm}$, which meant that the catheter had sufficient flexibility. The goal of the kink modeling was to decide whether the target specification can be reached through a design modification of the catheter made of the existing material (carbothane), or, if in addition to the new design, a material with enhanced properties needs to be synthesized.

\subsection{Determining Elastic Properties of the Material}

The first step in the modeling was to determine elastic properties of the material, based on the available experimental data.

For polymer materials with significant elastic deformations, the correlation between stresses and deformations is expressed through specific energy of deformations $W$. In case of uniaxial tension, this dependence can be expressed as [20]

$\sigma=2 \cdot\left(\lambda_{1}^{2}-\frac{1}{\lambda_{1}}\right) \cdot\left(\frac{\partial W}{\partial I_{1}}-\frac{1}{\lambda_{1}} \frac{\partial W}{\partial I_{2}}\right)$

where

$\sigma$ - axial stress (tension),

$I_{1}=\lambda_{1}+\lambda_{2}+\lambda_{3}$ - the first invariant of the deformation tensor,

$I_{2}=\lambda_{1}{ }^{2} \lambda_{2}{ }^{2}+\lambda_{1}{ }^{2} \lambda_{3}{ }^{2}+\lambda_{2}{ }^{2} \lambda_{3}^{2}$ - the second invariant of the deformation tensor

$\lambda_{p}=1+\varepsilon_{p}$ - relative elongation in the direction $p, p=1,2,3$

$\varepsilon_{p}$ - strain in the direction $p$.

Elastic properties of the catheter material were determined based on the experimental data, from axial and radial tension tests. The experimental setup and boundary conditions are shown in Fig. (8a) for the radial test and Fig. (8b) for the axial test. Experimental and analytical (obtained by FEA) elastic curves are shown in Fig. (9a) for radial and Fig. (9b) for axial loading. As the analytical model for the mate- 
(a)

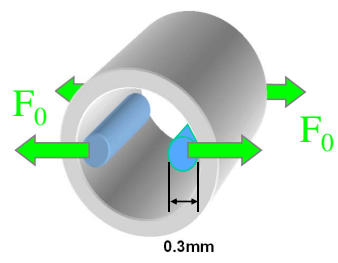

(b)

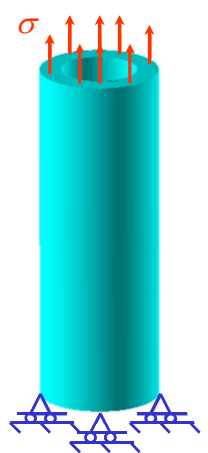

Fig. (8). Tests to determine constants in the material model. Radial (a) and axial (b) loading.
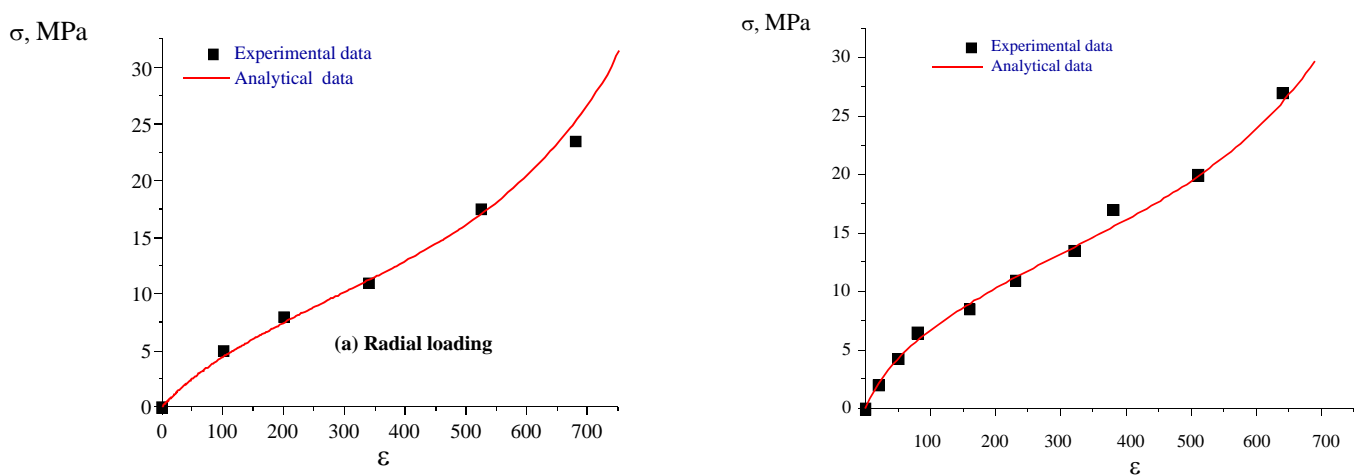

Fig. (9). Material model. Experimental data vs. analytical model.

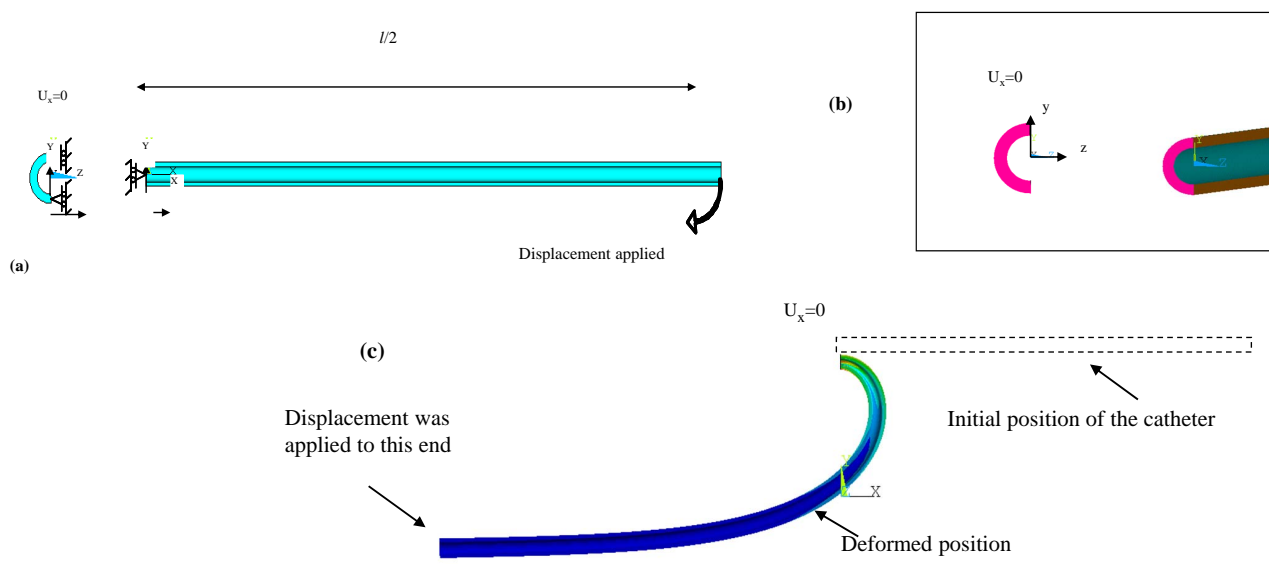

Fig. (10). Boundary conditions for the kink test modeling. (a, c): applying displacement at the right end, (a, b): boundary conditions, $U_{x}=0$ at the left end. $l$ is a length of the catheter, $\mathrm{U}_{\mathrm{x}}$ is a displacement along the $x$ axis. Only half of the cross-section, as shown in Fig. (b), was modeled.

rial, the Ogden two-parameter model [21] was selected. In this model, specific energy of deformation $W$ is expressed as

$W=\sum_{i=1}^{2} \frac{\mu_{i}}{\alpha_{i}}\left(\bar{\lambda}_{1}^{\alpha_{i}}+\bar{\lambda}_{2}^{\alpha_{i}}+\bar{\lambda}_{3}^{\alpha_{i}}-3\right)+\sum_{i=1}^{2} \frac{1}{d_{i}}(J-1)^{2 i}$

where

$J=\frac{V}{V_{o}} \quad$ - relative variation of volume

$\overline{\lambda_{p}}=J^{-1 / 3} \lambda_{p}, p=1,2,3$-relative elongation in the direction $p$

$\mu_{i}, \alpha_{i}, d_{i}$, - elastic constants that need to be determined, $i=1,2$
From the best fit to the experimental data (Fig. 9), the elastic constants were determined as follows:

\subsection{Results. Single Lumen Catheter Kink Resistance}

While bending stiffness and burst pressure are relatively easy to estimate, resistance to kink, which is discussed below, involves loss of stability (buckling) of the deformed shape and requires FEA modeling of a non-linear system with large deformations. Finite Element Analysis of the kink test was done both in ANSYS and ABAQUS.

Kink modeling was done for the cylindrical $40 \mathrm{~mm}$ long sample (which corresponds to half a length of the catheter), with the symmetrical boundary conditions at the left end (in the middle of the catheter) and displacement applied at the right end, as shown in Fig. (10). The kink was modeled as a non-linear quasi-static problem. Quadratic isoparametric 


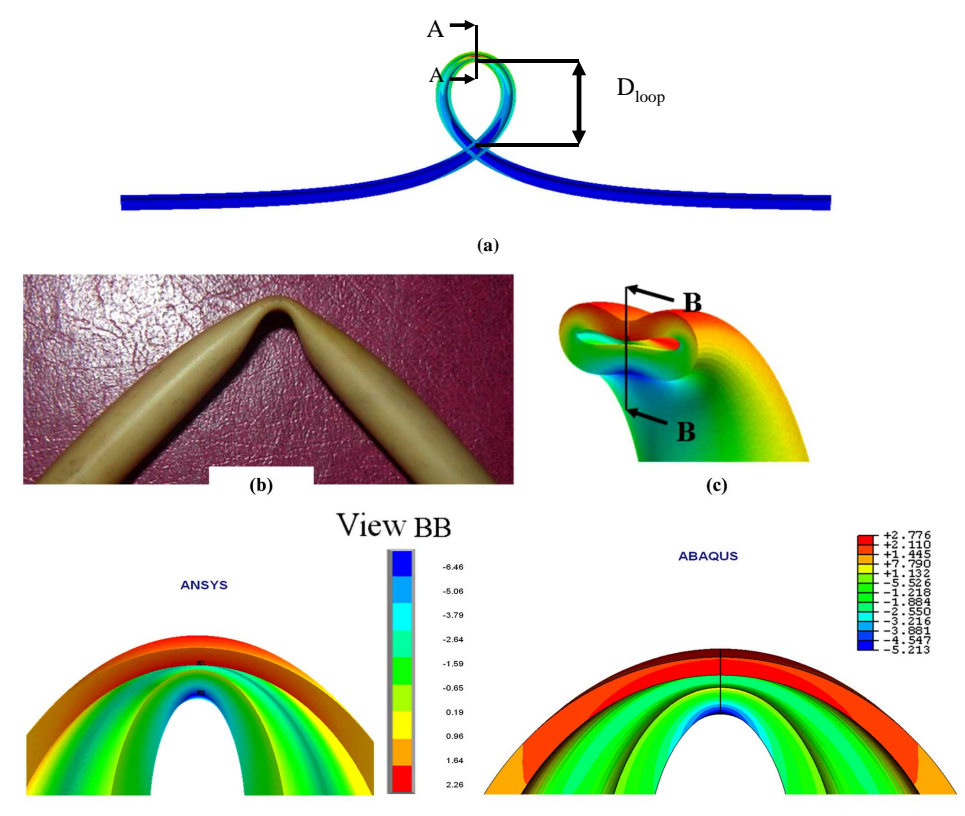

(d)

Fig. (11). Kink test modeling. (a): configuration of the catheter prior to buckling; further movement of tube ends leads to closing of crosssection AA. The experimental and analytical value for the loop diameter $\mathrm{D}_{\text {loop }}$ are $7.0 \mathrm{~mm}$ and $6.4 \mathrm{~mm}$; (b): photo of a kinked tube; (c): cross-section of the catheter after buckling; (d): comparison of results obtained in ANSYS and ABAQUS. The colors indicate intensity of axial stresses.

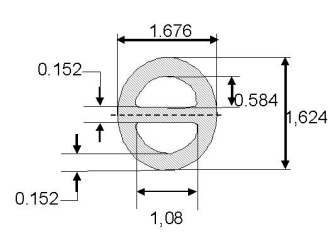

(a)

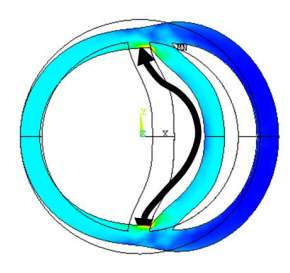

(b)

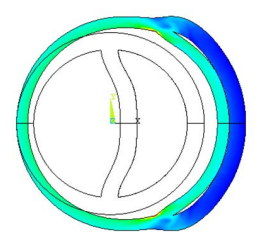

(c)

Fig. (12). Double lumen catheter. (a): dimensions $(\mathrm{mm}),(\mathbf{b})$ : determining the length of the partition, (c): catheter with the partition reaching the inner diameter.

spatial solid finite elements were used, the element size was $0.06 \mathrm{~mm}$. Convergence criteria, based on the residual forces and displacement vectors, were applied throughout FEA modeling.

The deformed shape of the catheter and the critical loop diameter (just prior to buckling) were calculated both in ANSYS and ABAQUS, and the value $6.4 \mathrm{~mm}$ for the critical loop, obtained in both packages, reasonably well agrees with the experimental value $(7.0 \mathrm{~mm})$ for the critical loop diameter for the catheter made of the same material. Both values are well within the specification, which requires that the critical loop be below $15.24 \mathrm{~mm}$. The deformed configurations of the catheter are shown in Fig. (11), where the colors indicate intensity of axial stresses.

To evaluate the reinforcement options, the effect of linear strengthening of the material on the loop radius was studied. Assuming the elastic characteristic follows the formula $\sigma=k$ $f(\varepsilon)$, where $f(\varepsilon)$ is the deformation diagram for the source material, the following data were obtained for the loop diameter:

$$
\begin{aligned}
& k=1 D_{\text {loop }}=6.4 \mathrm{~mm} \\
& k=10 D_{\text {loop }}=6.98 \mathrm{~mm}
\end{aligned}
$$

$$
\begin{aligned}
& \mathrm{k}=20 \mathrm{D}_{\text {loop }}=7.04 \mathrm{~mm} \\
& \mathrm{k}=50 \mathrm{D}_{\text {loop }}=7.23 \mathrm{~mm} \\
& \mathrm{k}=1000 \mathrm{D}_{\text {loop }}=10.34 \mathrm{~mm}
\end{aligned}
$$

This analysis shows that the critical diameter of the loop is relatively insensitive to the material stiffness, and therefore it was proposed to reinforce the material so that the catheter can pass both the kink and the pressure burst tests (Fig. 3). Considering different requirements to different parts of the catheter (Section 2.1), this reinforcement can be limited to the hub-taper section of the catheter.

\subsection{Results. Double Lumen Catheter. Pressure Resistance}

For the proposed double lumen catheter, we determined the critical pressure $\mathrm{p}^{*}$ under which the stretched folded partition will reach the inner diameter, closing the gap (Fig. 7); further growth of pressure would lead to growth of the outer diameter and therefore is not allowed). The FEA model (Fig. 12a) assumed that the partition is hanging freely, without creating any folds. The length of the partition $(l=1.35$ $\mathrm{mm})$ was calculated based on the given diameter of the catheter (Fig. 12b). Time-ramped pressure was applied in 


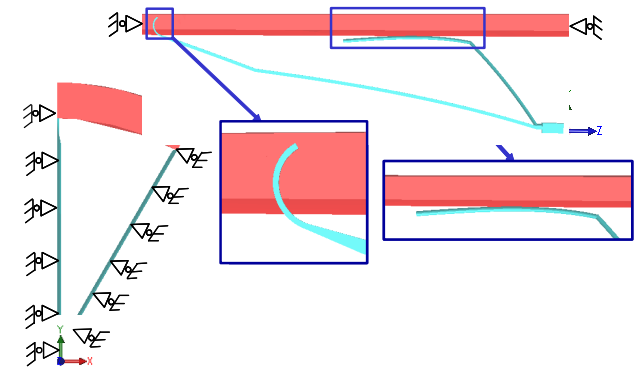

Fig. (13). Section of the filter and boundary conditions.
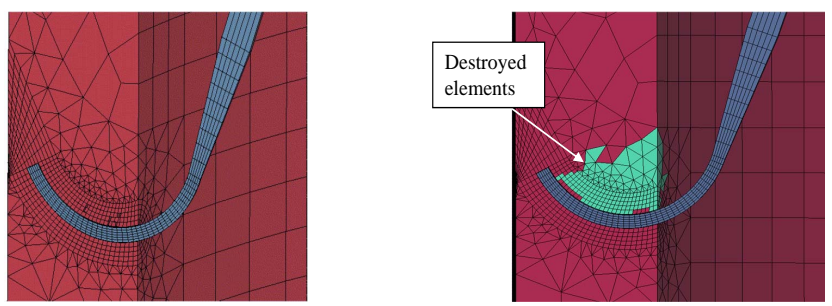

Fig. (14). Mesh in the wall around the hook (initial position, left, and deformed position, right, with shown destroyed finite elements).

one of the lumens. Possible multiple contact interaction (without friction) of the partition and the inner wall of the catheter was taken into account.

For the existing material, the critical pressure under which the stretched folded partition reaches the inner diameter (Fig. 12c) was determined $\mathrm{p}^{*}=123 \mathrm{psi}(848 \mathrm{kPa})$. At critical pressure, von Mises stresses at the juncture between the partition and the inner wall $(29 \mathrm{MPa})$ are still below the ultimate strength of carbothane (41.6-59.1 MPa) [22].

Like in the previous case, the effects of linear strengthening of the material on the critical pressure $\mathrm{p}^{*}$ was studied. Assuming the elastic characteristic following the formula $\sigma$ $=k f(\varepsilon)$, where $f(\varepsilon)$ is the deformation diagram for source material, the following values of the critical pressure $\mathrm{p}^{*}$ were obtained:

$$
\begin{array}{ll}
\mathrm{k}=2.0 & \mathrm{p}^{*}=215 \mathrm{psi}(1,482 \mathrm{kPa}) \\
\mathrm{k}=2.5 & \mathrm{p}^{*}=300 \mathrm{psi}(2,068 \mathrm{kPa}) \\
\mathrm{k}=3.0 & \mathrm{p}^{*}>300 \mathrm{psi}(2,068 \mathrm{kPa})
\end{array}
$$

Therefore, we come to a conclusion that in order to withstand the targeted pressure, $300 \mathrm{psi}=2,068 \mathrm{kPa}$, without large deformations of the catheter and without exceeding the ultimate strength, a material with enhanced properties is needed [1,3]. A polymer having the elastic characteristic (the $\sigma-\varepsilon$ curve) as that of carbothane multiplied by a factor of 2.5 makes it possible to double the cross-section of the double lumen catheter, without reaching the inner diameter. Maximum stresses in the stiffer material $(\mathrm{k}=2.5)$ do not reach the ultimate strength of carbothane.

\section{RESULTS OF FEA MODELING. RETRIEVABLE BLOOD CLOT FILTERS: MINIMIZING DAMAGE TO THE VEIN WALL}

A significant complication, prevention of which is discussed below, is difficulty of filter removal, due to in-growth (endothelialization) of the filter.
While a few solutions to the listed problem were generated through resolving the respective contradictions, similarly to what was described above, we focus below on Finite Element Analysis and optimization of the retrieval process, aimed at reducing the damage to the vein wall.

FEA modeling for a retrievable filter was aimed at optimization of the anchors in such a way as to minimize the threshold force (applied from the catheter-based retrieval device), required to start movement of the filter with respect to the vein wall (when the locators, Fig. (1b), start slipping relative to the wall). The damage to the wall in the model, estimated based on the number of destroyed finite elements, increased with the threshold force, and therefore the latter was taken as a proxy to estimate the vein damage.

Two scenarios of the filter removal were considered, static (under constant force) and kinematic (at constant speed). The non-linear, non-stationary problem with partial destruction of the mesh (the vein wall) was modeled in LSDYNA.

The elastic parameters of the vein were taken from [23], for a person 40-49 years old. The vein was modeled as an isotropic shell, with the Young modulus $\mathrm{E}=0.7 \mathrm{GPa}$, Poisson coefficient $\mu=0.45$, density $r=550 \mathrm{~kg} / \mathrm{m}^{3}$. Subsequent modeling has shown limited effect of the vein stiffness (Young modulus) on the threshold force.

The retrievable filter (Fig. 1b) includes locators, which position the filter in the vein, and anchors with the deformable hooks, which can unbend (stretch) and release the filter from the vein when the former is pulled out. Because of axial symmetry, only a $30^{\circ}$ section of the filter was modeled (Fig. 13), with the axial force, applied to the section along $Z$ axis, equal $1 / 12$ of the total force applied to the hub of the filter. In the model, the axial force was time-ramped, until the moment when the locators started moving with respect to the vein wall. An explicit time integration scheme with a step size $10^{-7} \mathrm{sec}$, based on the central difference method, was used. The mesh included 15,415 linear isoparametric spatial elements and 9,030 nodes. The element size in the area of contact between the hook and the vein was $0.01 \mathrm{~mm}$.

Multiple contact interaction spots between the locators and the wall were considered to define the contact area and simulate the forces acting between the filter and the vein walls more accurately. The Coulomb friction coefficients were taken as $\mu_{\text {vein-steel }}=0.1$ [24], $\mu_{\text {steel-steel }}=0.2$.

The considered factors, some of which are shown in Table 3, included combinations of pre-stressed (due to deformation of the locators in contact with the vein walls) and non-pre-stressed conditions of the filter. The results in these two cases were close. The dynamic effects (taking into account inertial forces acting on the vein walls), showed greater, but still limited, impact. The most important factor was the scenario of the removal process, which can take place either at a constant speed or under a growing force.

The models also took into account destruction of the vein wall, taking place as the hooks were unbent and removed from the walls. A critical value of the principal deformation $\varepsilon_{1}^{*}=80 \%$ was considered as a destruction criterion. Upon destruction, the respective finite elements were removed from the model (Fig. 14). 
Table 3. The Threshold Force Under Different Removal Scenarios

\begin{tabular}{|c|c|c|c|c|c|}
\hline Threshold Force & \multicolumn{3}{|c|}{ Time-Ramped Force } & \multirow{2}{*}{\multicolumn{2}{|c|}{$\frac{\text { Filter Moving at Constant Speed } 30 \mathrm{~cm} / \mathbf{s e c}}{\text { Not pre-stressed filter }}$}} \\
\hline \multirow[t]{4}{*}{ Fthr, $\mathrm{N}$} & Pre-stressed filter & \multicolumn{2}{|c|}{ Not pre-stressed filter } & & \\
\hline & Without dynamic effect & \multicolumn{2}{|c|}{ With dynamic effect } & \multicolumn{2}{|c|}{ With dynamic effect } \\
\hline & \multicolumn{3}{|c|}{ Without mesh destruction } & $\begin{array}{l}\text { Without mesh } \\
\text { destruction }\end{array}$ & With mesh destruction \\
\hline & 0.91 & $0.89^{*}$ & 1.2 & 7.3 & 8.0 \\
\hline
\end{tabular}

Additionally, sensitivity of the results to stiffness of the vena cava was estimated to be relatively low. For instance, for the lower right cell in Table $\mathbf{3}$ (Removal at constant speed, Not pre-stressed filter, Dynamic effect, Destruction of mesh), reducing vein stiffness by a factor of 50 increases the threshold force from 8.0 to $8.8 \mathrm{~N}$. The increase is primarily due to greater damage to the vein at lower stiffness.

Fig. (15) shows the filter with optimized geometry of the hook, which is almost totally unbent upon removal. Since the force calculated for removal with constant speed (typical for performance of a radiologist) is, in the beginning of the process, significantly higher, than in the removal under constant force scenario, the constant speed regime was used to estimate the vein damage.

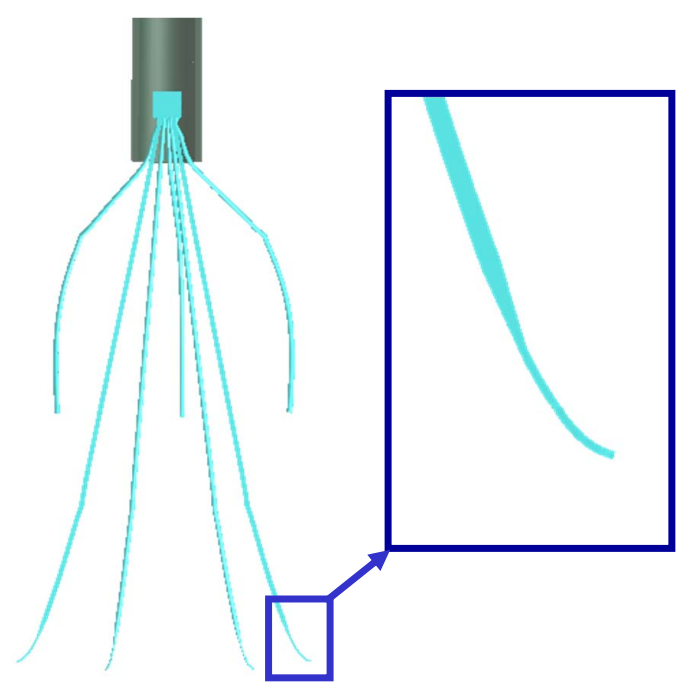

Fig. (15). The filter after removal from the wall. The hook is unbent, minimizing damage to the wall.

The proposed optimized design of the hook allows to significantly reduce damage done to the vein wall during the removal process.

\section{CONCLUSIONS}

The characteristics of prospective medical devices can be significantly improved by resolving contradictory requirements to their design. This approach makes it possible to meet challenging specifications which may be hard to achieve by using the traditional compromise-based design methods. In the presented examples, the target characteristics included maximum pressure of the peripherally inserted central catheter (increased up to $300 \mathrm{psi}=2,068 \mathrm{KPa}$ ) and maximum flow rate through a double lumen catheter (increased 16-fold due to effective doubling of the radius).
Once the designs are developed, advanced FEA modeling, which takes into account non-stationary non-linear deformations, mesh destruction and other factors, plays an important role in validation of the developed designs, due to difficulties in obtaining experimental data. FEA models are discussed for modeling a kink test of the catheter and the process of removal of a retrievable blood clot filter from the vena cava. In the latter case, sensitivity of the model to different factors, affecting the results, was studied, and the factors to which the results are most sensitive were identified.

\section{ACKNOWLEDGMENT}

The authors are grateful to the anonymous reviewers for their comments and suggestions.

\section{CONFLICT OF INTEREST}

None declared.

\section{REFERENCES}

[1] Bell BA, Elliott CJ, Dicarlo PD, et al. Medical Devices. US Patent Application 2008/0097350, April 2008.

[2] DiCarlo PD, Lareau R, Bell B, et al. Medical Devices. US Patent Application 2007/0135751, June 2007.

[3] Voznyakovski AP, Malinin L, Koblents PY, et al. Nano Particle Additives for Venous Access Catheter. US Patent Application 2008/0086096, April 2008.

[4] Malinin L, Kudryavtsev AV, Minaker VE, et al. Ultrasound Catheter. US Patent Application 2008/0097316, April 2008

[5] Yap YS Karapetis C, Lerose S, Iyer S, Koczwara B. Reducing the risk of peripherally inserted central catheter line complications in the oncology setting. Eur J Cancer Care 2006; 15(4): 342-349.

[6] Ng PK, Ault MJ, Ellrodt AG, Maldonado L. Peripherally inserted central catheters in general medicine. Mayo Clin Proc 1997; 72(3): 225-33.

[7] Lam S, Scannell R, Roessler D, Smith, MA. Peripherally inserted central catheters in an acute-care hospital. Arch Inten Med 1994; 154(16): 1833-7.

[8] SYNERGYTM CT PICC [homepage on the Internet]. Centerville, UT: Health Line International Corporation [cited: $25^{\text {th }}$ Oct 2011]. Available from http://www.hlic.net/component/content/article/102synergy-ct-picc.html

[9] Morpheus® CT Peripherally Inserted Central Catheter [brochure on the Internet]. Available from http://www.an-giodynaics.com/uploads/pdf/Peripherally\%20Inserted\%20Cent-ral-\%20Catheter.pdf [cited: $25^{\text {th }}$ Oct 2011].

[10] Pressure Injectable PICC Product [brochure on the Internet].. available from http://www.teleflex.com/en/usa/pro-ductAreas/vascularAccess/productGroups/picc/prod-ucts/Arrowev-olution/documents/picc-instructions-for-use.pdf [cited: $25^{\text {th }}$ Oct 2011]

[11] $510(\mathrm{k})$ Summary [document on the Internet]. Available from http://www.accessdata.fda.gov/cdrh_docs/pdf7/K-072625.pdf [cited: $25^{\text {th }}$ Oct 2011].

[12] Carr RM Jr, Chanduszko AJ, McDermott JD, Kaufman JA. Removable Embolus Blood Clot Filter. US Patent Application 2007/0112373, May 2007.

[13] Datta I, Ball CG, Rudmik L, Hameed SM, Kortbeek JB. Complications related to deep venous thrombosis prophylaxis in trauma: a 
systematic review of the literature. $\mathrm{J}$ Trauma Manag Outcomes, $6^{\text {th }}$ Jan. 2010 [cited: 25 ${ }^{\text {th }}$ Oct 2011]. 4:1. Available from http://www.traumamanagement.org/content/4/1/1

[14] Smouse B, Johar A. Is market growth of vena cava filters justified? Endovasc Today, Feb. 2010 [cited: $25^{\text {th }}$ Oct 2011]. Available from http://bmctoday.net/evtoday/pdfs/et0210_feature_smouse.pdf

[15] Gallieni M, Pittiruti M, Biffi R. Vascular Access in Oncology Patients. CA Cancer J Clin 2008; 58(6): 323-46.

[16] Ladewig GR. TRIZ: the theory of inventive problem solving. In: Griffin A, Somermeyer S, Eds. The PDMA ToolBook 3 for New Product Development, Hoboken, NJ: Wiley. 2007; pp. 3-40.

[17] Guo X, Johnson DP, Stehr R. Biocompatible polycarbonate and radiopaque polymer compositions and methods of manufacturing medical devices with same. US Patent Application 2010/0168270, July 2010

[18] U.S. Department of Health and Human Services. Guidance for Industry and FDA Staff. Class II Special Controls Guidance Document for Certain Percutaneous Transluminal Coronary Angioplasty (PTCA) Catheters [document on the Internet]. Available from http://www.fda.gov/MedicalDevic-es/DeviceRegulationandGuidance/GuidanceDocuments/ucm225145.htm [cited: $25^{\text {th }}$ Oct 2011].

[19] Salis AI, Eclavea A, Johnson MS, Patel NH, Wong DG, Tennery G. Maximal flow rates possible during power injection through currently available PICCs: an in vitro study. J Vasc Interv Radiol 2004; 15(3): 275-81

[20] Simo JC, Hughes TJR. Computational Inelasticity. Corrected edition. Berlin and Heidelberg: Springer-Verlag 1997.

[21] Ogden R.W. Nonlinear Elastic Deformations. Mineola, NY: Dover Publications 1984

[22] Materials and Coatings for Medical Devices: Cardiovascular. ASM International, 2009 [document on the Internet]. Available from www.asminternational.org [cited: $25^{\text {th }}$ Oct 2011]

[23] Begun PI, Shukeylo YuA. Biomechanics. St. Petersburg: Polytechnica 2000 (in Russian)

[24] Pate JW, Melvin D, Cheek RC. A New Form of Vena Caval Interruption. Ann Surg 1969; 169(6): 873-80.

Received: October 26, 2011

(C) Malinin et al.; Licensee Bentham Open.

This is an open access article licensed under the terms of the Creative Commons Attribution Non-Commercial License (http://creativecommons.org/licenses/by-nc/3.0/) which permits unrestricted, non-commercial use, distribution and reproduction in any medium, provided the work is properly cited. 